\title{
PREPARATION AND EVALUATION OF \\ CIPROFLOXACIN/CHITOSAN IMPLANTS AS A CONTROLLED BIODEGRADABLE DRUG DELIVERY SYSTEM
}

Eman S. El-Leithy

Department of Pharmaceutics, Faculty of Pharmacy, Helwan University

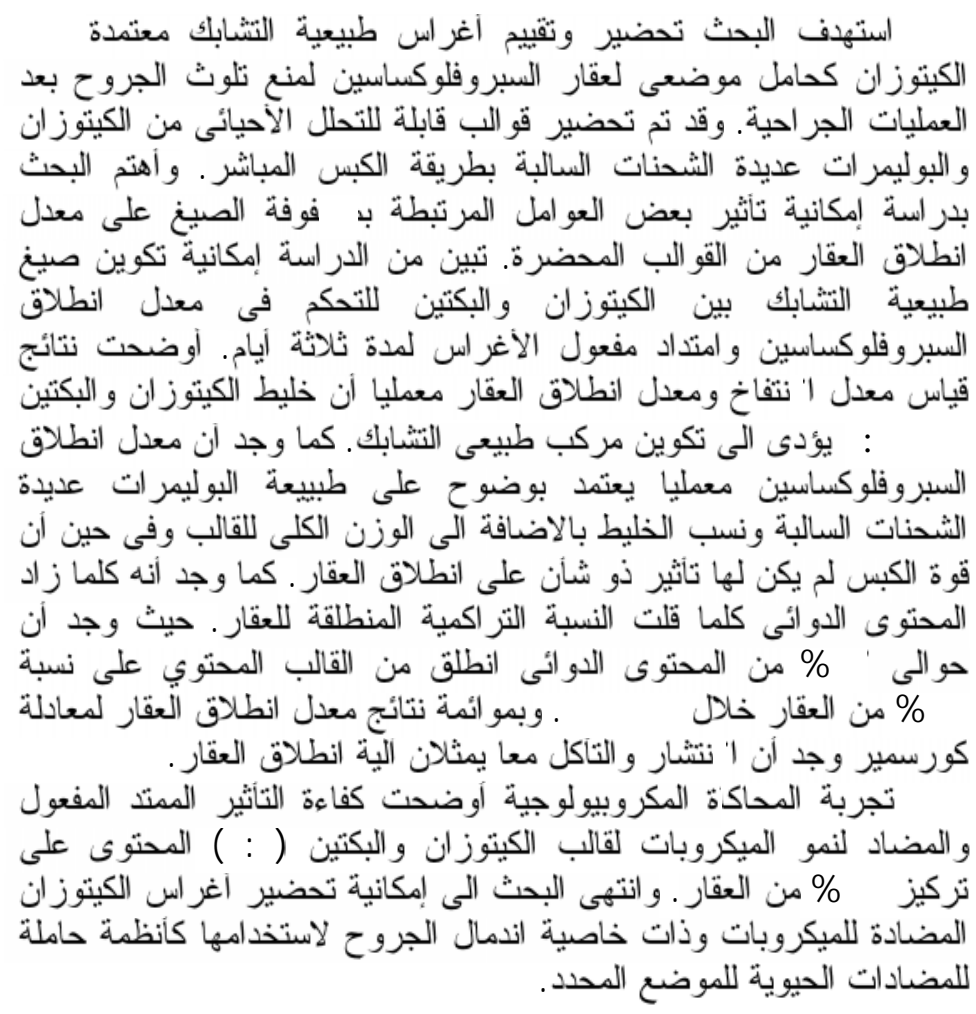

This work aimed to prepare and evaluate physically crosslinked implantable matrices based on chitosan as a site-specific delivery of ciprofloxacin hydrochloride (CFX.HCl) for the prevention of post-surgical wound infection. Biodegradable matrices were prepared from chitosan and different polyanion polymers by direct compression. The potential of formulation 
variables on the in-vitro drug release from polymeric matrices has been studied. A matrix system of CFX.HCl based on the polyanion complex of chitosan and pectin showed a sustainable drug release over three days. The swelling and in-vitro drug release experiments suggested that complex formation between chitosan and pectin has occurred at ratio 1:2. The results also suggested that CFX. $\mathrm{HCl}$ release from chitosan matrices was markedly influenced by the nature of polyanion polymers, mixing ratio and total weight of matrices, whereas the compression force did not significantly affect the drug release. With increasing CFX.HCl loading, a decrease of the percent cumulative release was observed. Approximately 13\% of loaded drug was released from matrix containing $25 \% \mathrm{w} / \mathrm{w}$ drug during 72 hrs in phosphate buffer $p H$ 7.4. Fitting the in-vitro drug release data to Korsmeyer equation indicated that combined effect of diffusion and polymer chain relaxation could be the mechanisms of drug release. Microbiological simulated test revealed the efficient microbial inhibitory effect of chitosan: pectin (1:2) matrix with $25 \%$ drug load over extended period of time.

In conclusion: Cross-linked chitosan implantable device for septic wounds or post surgical treatment with antimicrobial capability and wound healing properties was developed to serve as a site-specific delivery system for antibiotics.

\section{INTRODUCTION}

Ciprofloxacin is one of the third generation fluoroquinolones with a broad spectrum antibacterial activity and good penetration into most tissues $^{1 \& 2}$. It is currently available as tablet, parenteral and ophthalmic formulations. It has a plasma half-life of 3-5 hrs and distribute through many body fluids and tissues in therapeutic levels ${ }^{3}$, Ocular ${ }^{4}$, nasal ${ }^{5}$ and topical ${ }^{6}$ formulations have been investigated. Different dosage forms such as microspheres ${ }^{7}$ microparticles $^{8}, \quad$ nanoparticles ${ }^{9 \& 10}$, liposomes $^{11}$ and implants ${ }^{12 \& 13}$ of this antibiotic have been investigated.
Polymers such as alginate (ALG), pectin (Pe), carboxymethylcellulose (CMC) and chitosan (CS) have been described as biocompatible, biodegradable and mucoadhesive, enabling numerous pharmaceutical and biomedical applications ${ }^{14-18}$ including the design of controlled release devices.

Chitosan is a polysaccharide with a structure comparable to cellulose. CS and cellulose are both made from liner $\quad \beta(1 \rightarrow 4)$-linked monosaccharide. The primary amino groups lead to special properties, that render CS very interesting for pharmaceutical applications. Drug release formulations using chitosan matrix that has been reticulated covalently or ionically for the amino groups of the 
biopolymer have been described ${ }^{19 \& 20}$. Chitosan is also known in the wound management field for its haemostatic properties. Furthermore, it possesses other biological activities and affects macrophage function that helps in faster wound healing ${ }^{21}$. It also has the ability to stimulate cell proliferation, facilitate rapid wound reepithelialization and the regeneration of nerves within a vascular dermis ${ }^{22}$.

Systemic treatment with antibiotics requires high serum concentration for extended periods, with a greater incidence of side-effects, an increased cost and low patient compliance. The development of a controlled release system for ciprofloxacin is very interesting for postsurgical prophylaxis, prevention and treatment of infection. The following study aimed to develop a local delivery system containing ciprofloxacin hydrochloride (CFX.HCl) taking into consideration the wound healing properties of the selected polymers (alginate, pectin, carboxymethylcellulose and chitosan) as additive properties to the antibiotic activity. The drug release was studied as a function of formulation parameters such as the type of natural polyanion polymers, the chitosan/ polyanion ratios, drug loading, tablets weight and compression force, to determine the most appropriate formulation with a steady release over several days. The formulations were characterized using in-vitro release study and swelling properties. The effect of concomitant variations of these parameters on the release kinetics was also analyzed. Microbiological test was carried out to prove the efficacy of the proposed matrix as a site specific delivery system.

\section{MATERIALS AND METHODS}

\section{Materials}

High molecular weight chitosan (CS) and citrus pectin (Pe) were obtained from Sigma- Aldrich Chemie $\mathrm{GmbH}$, packed in Switzerland. Carboxymethylcellulose sodium salt (CMC) was purchased from Sigma Chemical Co., USA. Sodium alginate (ALG) was obtained from S.D. Fine-Chem. Ltd. Ciprofloxacin hydrochloride (CFX.HCl) was received from Misr For Medicinal Product Company, Egypt. All other reagents were of analytical or pharmaceutical grades.

\section{Methods \\ Preparation of drug implants}

Mixtures of chitosan with different polyanions were examined as vehicles for sustained release implants of ciprofloxacin. Tablet matrices (model drug carrier systems) were prepared by direct compression according to the method described by Remunan-Lopez et.al. ${ }^{23}$. The drug/ polymer mixture was prepared by homogenously mixing of the drug, CS and the polyanion polymer (ALG, Pe or $\mathrm{CMC}$ ) with efficient trituration in a ceramic mortar for 15 minutes. This method greatly improves the compaction properties of CS and the polyanion polymers. Hundred $\mathrm{mg}$ of the mixture was compressed at a 
pressure of 2 tons for one minute (IR 15- tons Specac hydraulic press (PerkinElmer, USA)). The compressed tablet matrices were easily removed from the die with no need for lubricant. The tablet weight and the compression force were increased to $200 \mathrm{mg}$ and 5 tons, respectively, for studying the effect of matrix weight and compression force as formulation variables. Composition of the prepared matrices is presented in Table 1.

\section{Swelling studies}

Swelling of the prepared tablets in phosphate buffer saline (PBS) $\mathrm{pH} 7.4$ was determined gravimetrically ${ }^{23}$. Tablets were placed on a previously weighed stainless steel support. The dried samples weights were measured directly. The supports and tablets were introduced into beakers containing $20 \mathrm{ml}$ of phosphate buffer ( $\mathrm{pH} 7.4)$ and placed in an incubator (FTC 90E Refrigerated incubator, made in Europe) at $37^{\circ} \mathrm{C}$. At predetermined time intervals, the devices were removed from the medium with the aid of a pair of forceps, carefully blotted with cleansing tissues to remove excess water and immediately weighed. The measurements were done in triplicate. The water uptake was calculated from the relative weight gain and the swelling ratio (SR) was expressed as:

$\mathrm{SR}=(\mathrm{Wt}-\mathrm{Wo}) / \mathrm{Wo}_{0}$

Eq. (1)

Where Wo is weight of dry tablet $(\mathrm{g})$ and $\mathrm{Wt}$ is weight of tablet at time $\mathrm{t}$ (g).

Table 1: Investigated formulae of ciprofloxacin hydrochloride-loaded implantable tablet matrices.

\begin{tabular}{||c|c|c|c|c|c||}
\hline $\begin{array}{c}\text { Formulae } \\
\text { No. }\end{array}$ & $\begin{array}{c}\text { Drug \% } \\
\text { (w/w) }\end{array}$ & $\begin{array}{c}\text { Polymers } \\
\text { Composition }\end{array}$ & $\begin{array}{c}\text { Ratio } \\
\text { W/W }\end{array}$ & $\begin{array}{c}\text { Tablet } \\
\text { Weight } \\
\text { (mg) }\end{array}$ & $\begin{array}{c}\text { Compression } \\
\text { Force } \\
\text { (tons/minute) }\end{array}$ \\
\hline F1 & 2.5 & CS: ALG & $1: 3$ & 100 & 2 \\
\hline F2 & 2.5 & CS: CMC & $1: 3$ & 100 & 2 \\
\hline F3 & 2.5 & CS: Pe & $1: 3$ & 100 & 2 \\
\hline F4 & 2.5 & CS: Pe & $1: 2$ & 100 & 2 \\
\hline F5 & 2.5 & CS: Pe & $1: 1$ & 100 & 2 \\
\hline F6 & $\mathbf{5}$ & CS: Pe & $1: 2$ & 100 & 2 \\
\hline F7 & $\mathbf{1 0}$ & CS: Pe & $1: 2$ & 100 & 2 \\
\hline F8 & $\mathbf{2 5}$ & CS: Pe & $1: 2$ & 100 & 2 \\
\hline F9 & 10 & CS: Pe & $1: 2$ & $\mathbf{2 0 0}$ & $\mathbf{5}$ \\
\hline F10 & 10 & CS: Pe & $1: 2$ & 100 & \\
\hline
\end{tabular}

CS, chitosan; CMC, carboxymethylcellulose; Pe, pectin; ALG, sodium alginate. 


\section{In-vitro release studies}

Release from tablet matrices was performed through semipermeable cellulose membrane $(5 \times 5 \mathrm{~cm}$ Spectrapor membrane tubing No. 2 Spectrum medical industries, USA) wrapped around the tablets exposed to dissolution medium. Tablets were hanged inside the middle of beakers containing $50-\mathrm{ml}$ isotonic phosphate buffer saline (PBS, pH 7.4) and covered with paraffin film to minimize the rate of medium evaporation. Uniform mixing was accomplished using magnetic stirring $(50 \mathrm{rpm})$. All the experimental sets were placed inside incubator with controlled temperature $37 \pm 0.5^{\circ} \mathrm{C}$ for 3 days. At appropriate time intervals, $1 \mathrm{ml}$ sample was withdrawn and replaced with the same volume of fresh phosphate buffer. Every 24 hrs the release medium was slightly adjusted to $50 \mathrm{ml}$ before sample withdrawing. Drug absorbance was measured spectrophotometrically at $\lambda$ $273 \mathrm{~nm}$ after appropriate dilution (PerkinElmer Lambada EZ 201, Spectrophotometer, USA). The concentrations of CFX.HCl were calculated from the standard curve. Dissolution assays were carried out in triplicate for each sample.

\section{Kinetic analysis of the dissolution data}

To study the mechanism of the drug release from the matrices, release data were fitted according to Korsmeyer et.al. equation ${ }^{24}$, which is often used to describe the drug release behavior from polymeric systems. $\log (\mathrm{Mt} / \mathrm{M} \infty)=$

$$
\log \mathrm{k}+\mathrm{n} \log \mathrm{t} \quad \text { Eq. (2) }
$$

Where, Mt is the amount of drug released at time t; $\mathrm{M}_{\infty}$ is the amount of drug released after infinite time; $\mathrm{k}$ is a release rate constant incorporating structural and geometric characteristics of tablets; and $n$ is the diffusional exponent indicative of the mechanism of drug release.

To determine the release exponent, the log value of the fraction of released drug was plotted against log time according to Equation 2. The $\mathrm{n}$ value of 1 corresponds to zero-order release kinetics, $0.5<\mathrm{n}<1$ means a non-Fickian release model and $n=0.5$ indicates Fickian diffusion (Higuchi model) $)^{25}$.

Mean dissolution time (MDT) is a measure of the dissolution rate: the higher the MDT, the slower the release rate. MDT was calculated from dissolution data using Mockel and Lippold equation ${ }^{26}$ :

$$
\mathrm{MDT}=(\mathrm{n} / \mathrm{n}+1) \cdot \mathrm{K}^{-1 / \mathrm{n}} \quad \text { Eq. (3) }
$$

Where $\mathrm{n}=$ release exponent and $\mathrm{k}=$ release rate constant.

\section{Statistical analysis of the dissolution data}

Similarity $\left(f_{2}\right)$ and difference $\left(f_{1}\right)$ tests were used to compare CFX.HCl delivery profiles between different formulations ${ }^{27}$. The difference factor $\left(f_{1}\right)$ calculates the percent $(\%)$ difference between the two curves at each time point and is a measure of the relative error between the two curves: 
$\mathrm{f}_{1}=\left\{\left[\sum_{\mathrm{t}=1}{ }^{\mathrm{n}}\left|\mathrm{R}_{\mathrm{t}}-\mathrm{T}_{\mathrm{t}}\right|\right] /\left[\sum_{\mathrm{t}=1}{ }^{\mathrm{n}} \mathrm{R}_{\mathrm{t}}\right]\right\} \bullet 100$

where $n$ is the number of time points, $R_{t}$ is the dissolution value of the reference (prechange) batch at time $t$, and $T_{t}$ is the dissolution value of the test (postchange) batch at time $t$.

The similarity factor $\left(f_{2}\right)$ is a logarithmic reciprocal square root transformation of the sum of squared error and is a measure of the similarity in the percent (\%) dissolution between the two curves.

$\begin{aligned} f_{2}= & 50 \bullet \log \left\{\left[1+(1 / n) \sum_{t=1} n\right.\right. \\ & \left.\left.\left(R_{t}-T_{t}\right)^{2}\right]^{-0.5} \bullet 100\right\}\end{aligned}$

$\left.\left.\left(\mathrm{R}_{\mathrm{t}}-\mathrm{T}_{\mathrm{t}}\right)^{2}\right]^{-0.5} \cdot 100\right\} \quad$ Eq. $(5)$

For curves to be considered similar, $f_{1}$ values should be close to 0 , and $f_{2}$ values should be close to 100 . Generally, $\mathrm{f}_{1}$ values up to $15(0-15)$ and $\mathrm{f}_{2}$ values greater than 50 (50-100) ensure sameness or equivalence of the two curves and, thus, of the performance of the test (postchange) and reference (prechange) products. This model independent method is most suitable for dissolution profile comparison when three to four or more dissolution time points are available. Similarity and difference tests were used to compare $\mathrm{CFX} . \mathrm{HCl}$ delivery profiles between the different formulations.

\section{Microbiological evaluation of drug release in a gel simulating in-vivo implantation}

The method of Allababidi and $\mathrm{Shah}^{28}$ was adopted to study the in-vitro drug release from implantable matrices into agar gel. The release method was developed to simulate the in-vivo implantation conditions and predict the release characteristics from the implantable matrices when they are actually used in-vivo. Tablet matrices containing $10 \%$ and $25 \%$ w/w CFX.HCl have a weight of $100 \mathrm{mg}$ each and compressed at 2 tons for one minute were tested against ciprofloxacin-susceptible Staphylococcus aureus strain (grampositive organisms) and Escherichia coli (gram-negative organisms) compared to control. The agar crystals were dissolved in phosphate buffer $\mathrm{pH} 7.4$ to prepare $1.5 \%$ agar solution. Twenty gm of agar solution containing organisms were poured into Petri dish and left to congeal. A hole, equivalent in size to one tablet was made in the agar gel plate at the center with cork borer and the tablet was then implanted into the hole. Another twenty gm of agar solution containing organisms was poured on the top of the first layer containing the tablet and left to congeal. The plate was covered and placed in the incubator at $37^{\circ} \mathrm{C}$. The widths of the inhibition zones after $24 \mathrm{hrs}$ were used to estimate the antimicrobial activity corresponding to tested matrices with different drug concentrations. The release of ciprofloxacin from chitosan matrices into the surrounding gel was tested by subjecting samples $1 \mathrm{~cm}$ and $2 \mathrm{~cm}$ far from the end of inhibition zones for further microbial growth for $48 \mathrm{hrs}$. 


\section{RESULTS AND DISCUSSION}

\section{Evaluation of implants swelling}

The molecular structure of pectin, chitosan and ciprofloxacin hydrochloride is presented in Figure 1. Electrostatic interaction between polycationic polymer chitosan and different polyanion polymers leads to the formation of polyelectrolyte complexes (PECs) that control the drug release ${ }^{29}$. The degree of PECs formation was measured by the ability of tablets to gain weight and the results are presented in Figure 2. The results showed that the highest weight gain was observed with tablets composed of CS/ALG or CS/CMC (F1and F2). The lowest weight gain was observed with $\mathrm{CS} / \mathrm{Pe}$ tablets (F3). After 2 hrs of incubation in PBS pH 7.4, the CS/CMC and CS/Pe tablets kept their original shape with partial swelling, while CS/ALG tablets showed complete erosion after 60 minutes.

The results of F1, F2 and F3 showed pronounced increase in weight, due to water uptake and swelled up to 2.5, 2.4 and 1.3 times of their original weights, respectively, after $1 \mathrm{hr}$. The swelling of the matrices containing CS/ALG and $\mathrm{CS} / \mathrm{CMC}$ was more than those of $\mathrm{CS} / \mathrm{Pe}$ at $\mathrm{pH}$ 7.4. This result was related to the lower degree of crosslinking between the high percentage of carboxylic acid groups in alginate or CMC macromolecular chains and lower percentage of protonated positively charged amino groups on the chitosan molecules with pKa of $6.3^{30}$. This result showed that both anionic polymers (CMC and ALG) under the $\mathrm{pH}$ of the study, did not able form a complex, but behaved as inert soluble excipients. Consequently, upon diffusion of the release medium into CS/ALG and CS/CMC tablets, the water-soluble polymers ALG and CMC rapidly hydrated, swelled and dissolved. These results are in accordance with the work of Remunan-Lopez et.al. ${ }^{23}$.

The minimal swelling of F3 $(\mathrm{CS} / \mathrm{Pe})$ up to 1.3 times of their original weight after 2 hrs may be related to intermolecular $\mathrm{H}$-bonding between the $\mathrm{COOH}$ groups of pectin or $\mathrm{NH}_{2}$ groups of chitosan and $\mathrm{OH}$ or $\mathrm{COOCH}_{3}$ groups elsewhere within the matrix network (Fig. 1). The hydrogen bonding and hydrophobic forces between or within pectin molecules may also result in a tightening of network leading to reduced swelling capacity $^{31}$. Apparently, CS/Pe attractive forces were greater than repulsion forces that tend to decrease particles swelling. Such results showed that chitosan-pectin matrix could sustain the drug release in PBS pH 7.4. It has been previously reported that an implant with a high degree of swelling may modify host response and the drug release kinetics by the increased pressure on the surrounding tissue. Moreover, inflammatory cellular reactions may be modified by polymer hydrophilicity ${ }^{32}$

The effect of chitosan /pectin ratio on the swelling behavior was studied and results are presented in Figure 3. The swelling studies for $2 \mathrm{hrs}$ showed that tablets swell to different 
(a) chitosan

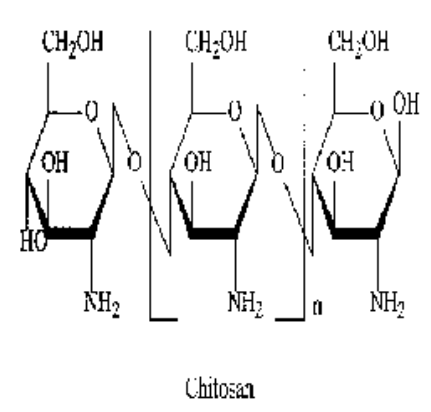

(c) Ciprofloxacin $\left[\mathrm{C}_{17} \mathrm{H}_{18} \mathrm{FN}_{3} \mathrm{O}_{3} \cdot \mathrm{HCl} \cdot \mathrm{H}_{2} \mathrm{O}\right]$ (b) Pectin
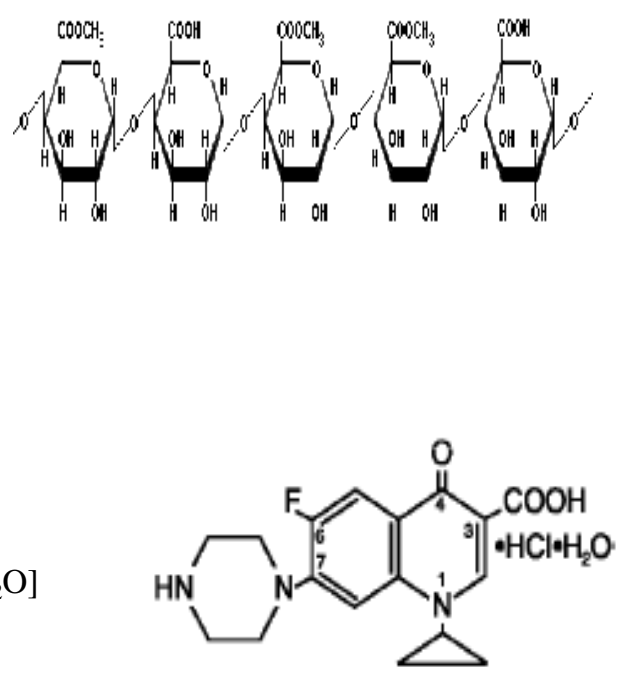

(e)
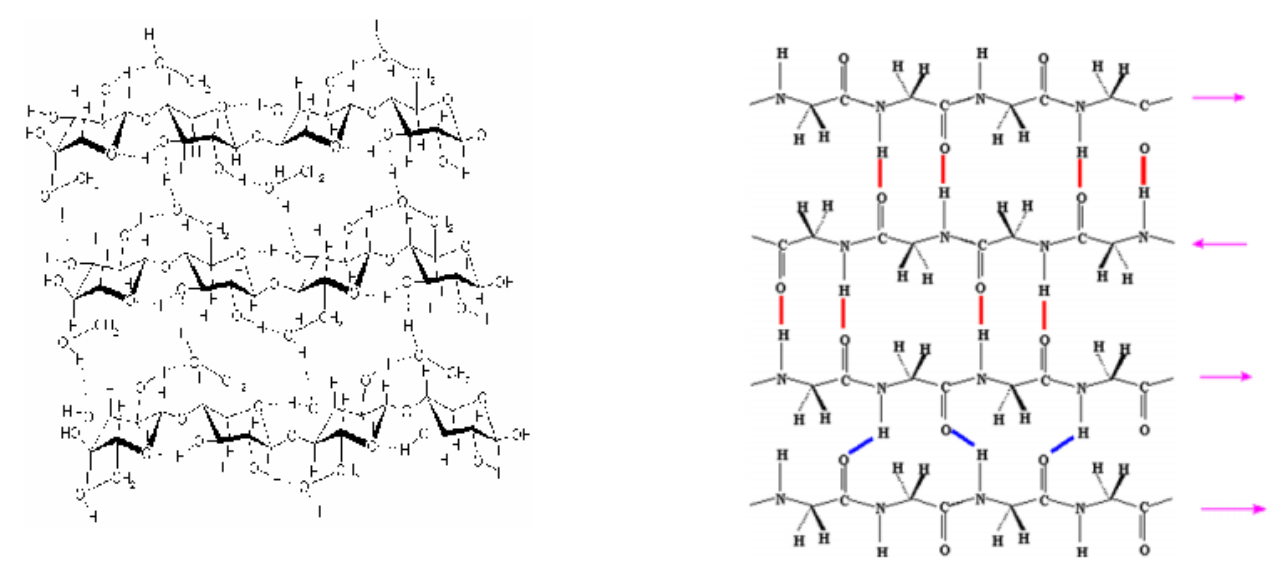

Fig. 1: Schematic representation of (a) chitosan, (b) pectin and (c) ciprofloxacin and the possible hydrogen bonds patterns between (d) pectin or (e) chitosan/ pectin molecules. 


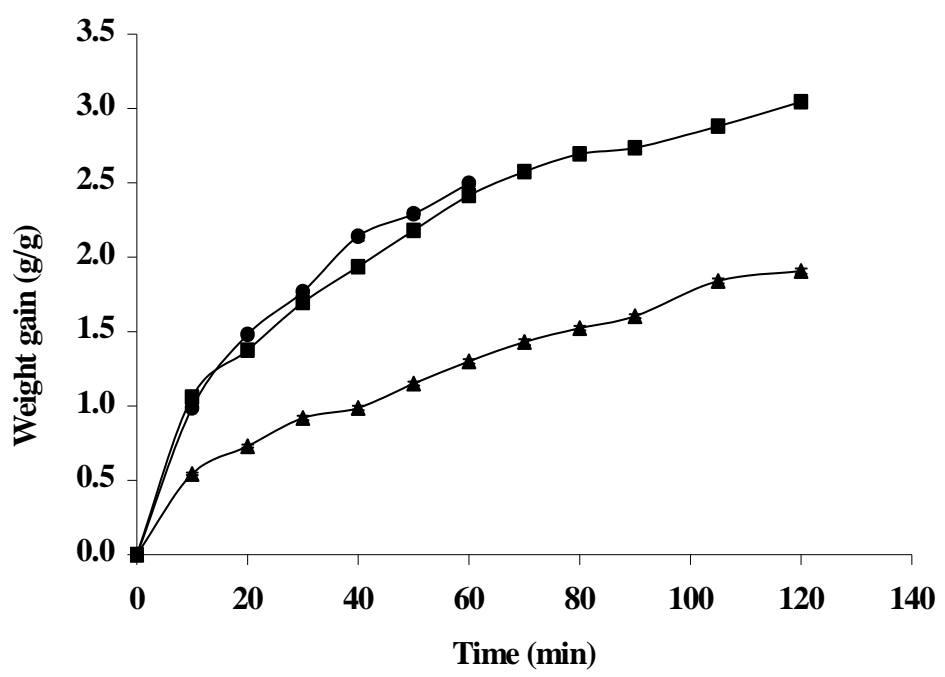

Fig.2. Swelling ratio of tablet matrices with different ployanion polymers at ratio 1:3 immersed in phosphate buffer $\mathrm{pH} 7.4$ at $37^{\circ} \mathrm{C}$.

$\rightarrow$ F1 (CS: ALG) $\rightarrow$ F2 (CS:CMC) $\leftarrow$ F3 (CS:Pe)

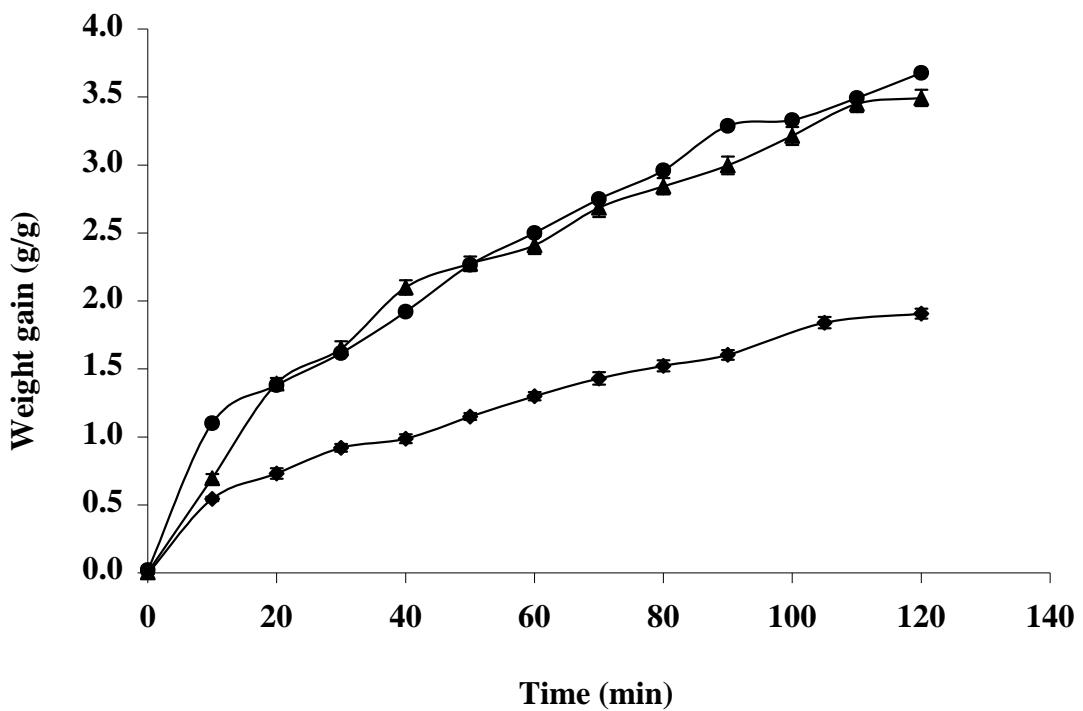

Fig. 3. Swelling ratio of tablet matrices with different ratios of Pectin immersed in phosphate buffer pH 7.4 at $37^{\circ} \mathrm{C}$.

$\multimap$ F3 (CS:Pe 1:3) $\rightarrow$ F4 (CS:Pe 1:2) $九$ F5 (CS:Pe 1:1) 
extents depending on the percentage of chitosan. Figure 3 shows that the weight gain was nearly similar and maximal with tablets F4 and F5 (CS: Pe 1:2 and 1:1 ratios, respectively) and minimal with F3 (CS: Pe 1:3). Swelling of the tablets can be explained by consideration of chitosan: pectin ratio, as well as the charges left by any excess of the two polymers. When chitosan was present in excess (F4 and F5) there was an excess of $\mathrm{NH}_{2}$ groups at higher $\mathrm{pH}$ values resulting in reduction of the electrostatic interaction (PECs) or cross-linking with highly ionized polyanions pectin. Therefore, increasing chitosan percentage may loose the PECs network and decrease the effect of intra-molecular $\mathrm{H}$ bonding between the $\mathrm{COOH}$ groups of pectin and $\mathrm{NH}_{2}$ groups of chitosan leading to increased tablets swelling capacity. With excess pectin F3 (CS: Pe 1:3) there was a greater possibility for intramolecular hydrogen bonds and hydrophobic forces within pectin molecules . Comparable conclusions were reached by Macleod et.al. ${ }^{29}$, who reported that optimal PEC formation would be expected to occur at $\mathrm{pH} 5.0$ when pectin: chitosan ratio lies somewhere between 2:1 and 3:1.

\section{In-vitro drug release studies}

Profiles of drug release from matrices composed of chitosan and anionic crosslinking polymer (ALG, $\mathrm{CMC}$, and $\mathrm{Pe}$ ) at ratio $1: 3$ and $2.5 \%$ drug loading are shown in Figure 4.
This figure reveals the differences in the release patterns as a function of polyanion type and the gradual diffusion of the drug from the swollen tablets. Approximately $80 \%, 71 \%$ and $59 \%$ of total drug were released after 24 hrs from F1, F2 and F3, respectively. Extending the drug release for $72 \mathrm{hrs}$ showed a slight increase in the percentage of drug released that ranged from $75 \%$ to $84 \%$ for F2 and F1, respectively. F3 showed a pronounced increase in the drug release reaching up to $71 \%$. These results showed that tablets containing pectin as polyanion polymer seemed to swell less and release the drug at lower rate in PBS $(\mathrm{pH}$ 7.4) as compared with tablets containing ALG and CMC. CS/Pe combination showed a greater tendency for complex formation at $\mathrm{pH} 7.4$ than CS/ALG or CS/CMC at the same $\mathrm{pH}$ value.

The effect of increasing chitosan ratio from CS: Pe 1:3 to 1:2 and 1:1 on the drug release profiles from chitosan tablets is presented in Figure 5. The overall percentage release of CFX. $\mathrm{HCl}$ after $72 \mathrm{hrs}$ was $71 \%, 61 \%$ and $68 \%$ for F3, F4 and F5, respectively. The results were contradictory to those obtained in swelling studies. It was found that increasing CS concentration F4 and F5 with CS: Pe ratio 1:2 and 1:1, respectively, lowered the drug release from tablet matrices than F3 (CS: Pe ratio 1:3). The results also revealed that F4 showed a more controlled 


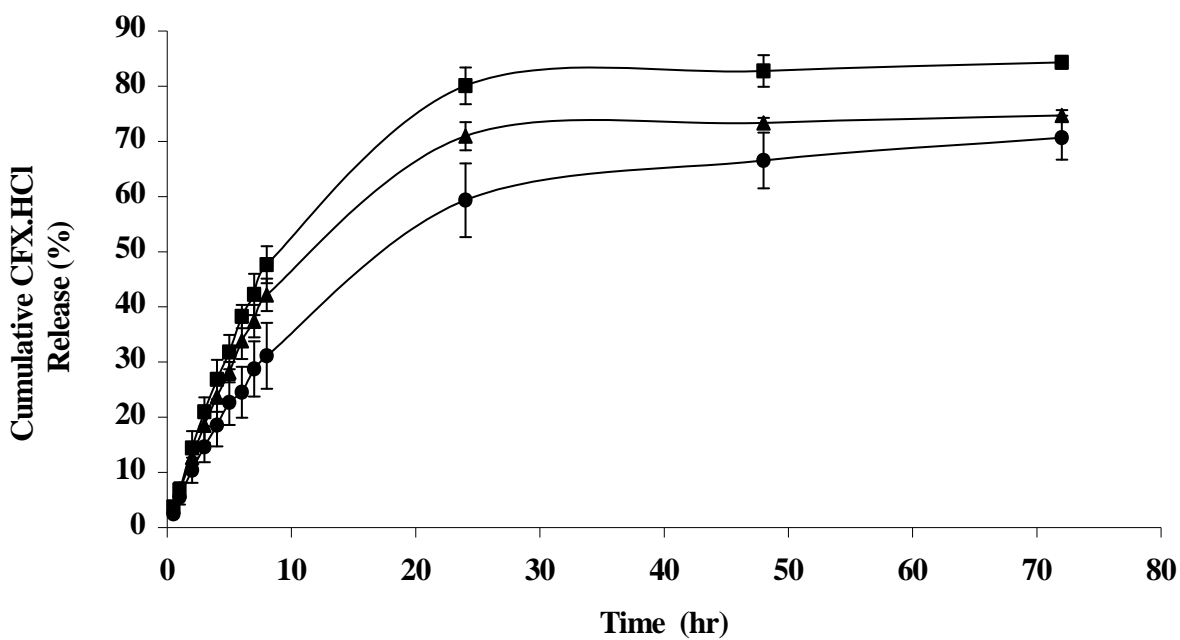

Fig. 4. Effect of polyanion polymers on the drug release from tablet matrices containing chitosan:polyanion polymers at ratio 1:3

$\rightarrow$ F1 (CS: ALG) $\quad \leftarrow$ F2 (CS:CMC) $\quad$ F3 (CS:Pe)

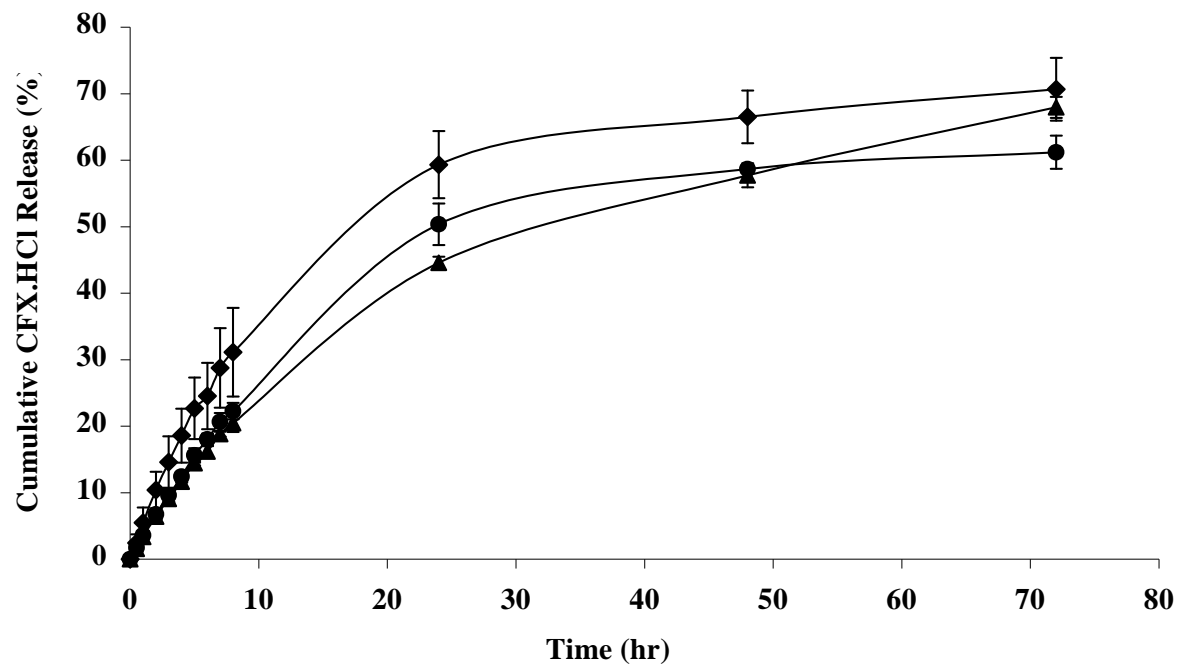

Fig. 5. Effect of chitosan: pectin ratio on the drug release from tablet matrices.

$\neg$ F3 $(1: 3)$

$\rightarrow$ F4 (1:2)

$\neg-F 5(1: 1)$ 
drug release. Such results may be related to the effect of the polymers ratio on the viscosity of the swelled tablets rather than PEC formation. Prolonged exposure of tablets to the dissolution medium increases water diffusion where the tablets did not have a suitable morphology but changed into gels with different viscosities according to ratios of polymers. Increasing the ratio of chitosan in the matrix increased gel viscosity that decreased drug diffusion from swelled gel. This is in accordance with the results of Chang and $\operatorname{Lin}^{33}$.

On the other hand, the slight increase in percentage drug released from $F_{3}$ may be related to further swelling and transformation of the tablets into gel forms after prolonged exposure (72 hrs) to PBS. Such transformation would be followed by a decrease in effect of hydrogen bonds and hydrophobic forces between or within pectin molecules, thus increasing the effect of the repulsive forces between fully negatively charged - COO- . Ghaffari et.al. ${ }^{34}$ reported that maximum yield of PEC formation between pectin and chitosan at $\mathrm{pH} 5.4$ was $2: 1 \mathrm{w} / \mathrm{w}$ ratio. In the present study, the formula containing chitosan: pectin ratio of $1: 2$ was selected for investigating the other formulation factors.

The effect of drug loading on delivery profiles was studied for tablet formulae F4, F6, F7 and F8. Results presented in Figure 6 revealed that drug release during the first 24 hrs was $50.37,34.17,20.37,7.84 \%$ and reached 61, 59, 37.3 and $13.3 \%$ after 72 hrs for F4, F6, F7 and F8, respectively. It has been shown that drug release decreased markedly with increasing drug concentrations, being more profound when drug concentration was $25 \%$. This finding is of interest since increasing the matrix loading can prolong release duration. Such results may be related to the possible effect of CFX.HCl loading on matrix $\mathrm{pH}$ upon the diffusion of the dissolution medium and bonds formation between the two polymer chains. Drug loading may result in a decrease in $\mathrm{pH}$ inside the matrix and increase the chance of existing chitosan as positively charged amino groups $\left(\mathrm{NH}^{+}\right)$that would increase the possibility of PEC ionic interaction $\left(\mathrm{NH}^{3+-} \mathrm{OOC}\right)$ between the two polymers, forming a closer network and decreasing drug diffusion outwards the matrix.

Another factor to be considered is the potential interaction between polymer and drug, which could affect drug diffusion through the matrix due to attraction or repulsion forces. A previous study of Mulhbacher et.al. ${ }^{35}$ demonstrated that the release of acetylsalicylic acid was slower from carboxylated crosslinked high amylose starch when the drug loading was increased. This was attributed to physical interactions between carboxylic groups of the drug and different interacting sites of polymers. The present study showed that a possible interaction between CFX.HCl, CS and Pe has been occurred (Fig. 3). 
Moreover, solubility and hydrophilicity of drug must be considered. At a high loading, water diffusion into the matrix might be altered. Désévaux et al. ${ }^{12}$ found that increasing CFX.HCl loading in crosslinked high amylose starch (CLHAS) matrix decreased the cumulative percent of CFX.HCl release in both 24-hrs and extended dissolution tests. Their results showed that water uptake into CLHAS matrix decreased inversely to drug loading regardless of matrix composition indicating the influence of the hydrophobic moiety of the CFX.

Figure 6 also showed a marked decrease in the total percentage of drug release from $50.37 \%(\mathrm{~F} 4)$ to $7.84 \%$ (F8) with increasing CFX.HCl loading 10 times (from $2.5 \%$ to $25 \%$ ). Therefore, chitosan implantable matrices with high drug loading $(25 \%$ or more) would be more advantageous to prolong release duration in-vivo and thus more appropriate for possible local antibacterial therapy.

To investigate the effect of matrix weight on the drug release from CS: Pe tablets at weight ratio $1: 2$, the tablets were prepared at two weights 100 and $200 \mathrm{mg}$ (F7 and F9), respectively. Figure 7 shows that drug release from the $100 \mathrm{mg}$ tablets was $37.35 \%$, while that from $200 \mathrm{mg}$ tablets was $19.19 \%$ during $72 \mathrm{hrs}$ release period. This indicates an inverse proportionality between tablet weight and the drug release. Doubling the tablet weight decreased the percentage drug released to the half.
The amount of CFX.HCl in 200 $\mathrm{mg}$ tablets was greater than that of $100 \mathrm{mg}$; hence the $\mathrm{pH}$ inside the matrix will be shifted to lower value with increasing the probability of polyelectrolyte complex formation between pectin and chitosan in the outer region of tablets upon diffusion of dissolution medium. Sustained release observed in the case of high tablet weight may be related to increase tablet thickness, formation of surrounding region of polyelectrolyte complex (PECs) network that control the drug diffusion and decreased obviously the initial drug release. Inner core of the tablets would not be able to fully hydrated with dissolution medium. In case of low tablet weight, matrix is thin, fully hydrated with dissolution medium and drug is released with higher percentage before the PECs network control drug diffusion.

Figure 8 shows the influence of compression force on drug release profiles from CS: Pe matrices at weight ratio 1:2. The tablets had total weight of $100 \mathrm{mg}$ and compression forces were 2 and 5 tons (F7 and F10). The results showed that increasing applied pressure influenced drug release rate and the percentage of drug release to a certain limit, whereas, there was a decrease in percentage of drug release from $37.35 \%$ (F7) to $30.9 \%$ ( F10) after 72 hrs release period with increasing the compression force from 2 to 5 tons. This result may be related to interparticulate friction between polymers particles, increasing the number of 


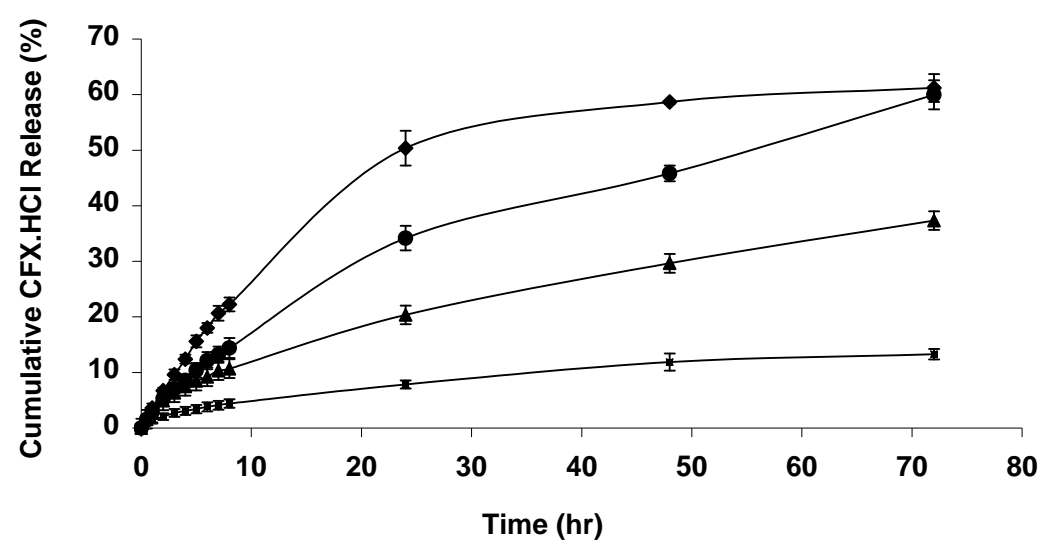

Fig. 6. Effect of drug loading on the drug release from tablet matrices with chitosan:pectin ratio 1:2

$\begin{array}{ll}\rightarrow \mathrm{F} 4(2.5 \% \mathrm{CFX} . \mathrm{HCl}) & \rightarrow \mathrm{F} 6(5 \% \mathrm{CFX} . \mathrm{HCl}) \\ \rightarrow \mathrm{F} 7(10 \% \mathrm{CFX} . \mathrm{HCl}) & \rightarrow \mathrm{F} 8(25 \% \mathrm{CFX} . \mathrm{HCl})\end{array}$

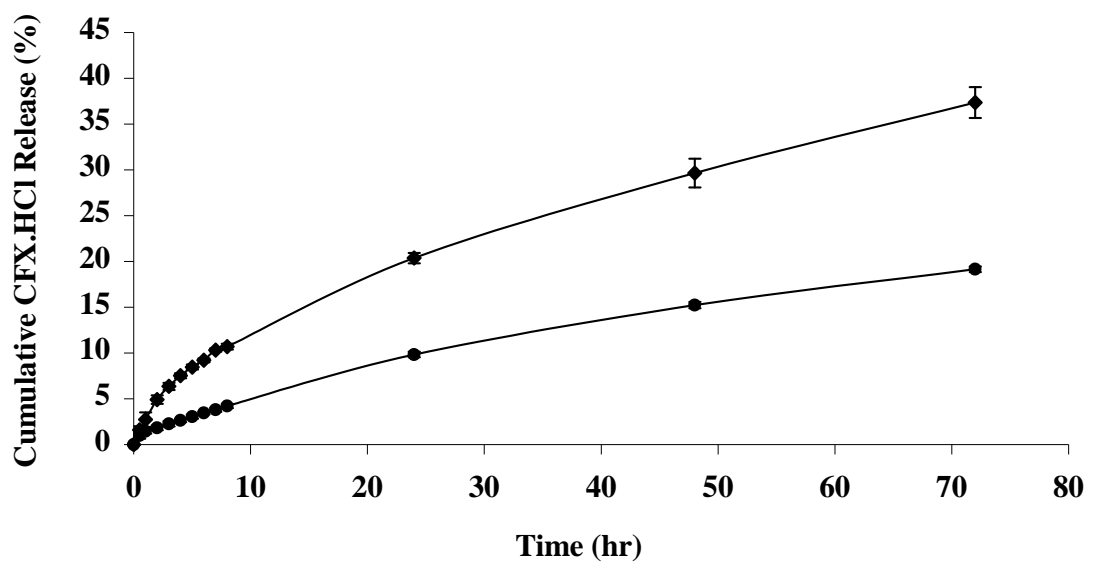

Fig. 7. Effect of matrix weight on the drug release from tablet matrices with chitosan:pectin ratio 1:2

$\multimap$ F7 (100 mg) $\quad \longrightarrow$ F9 (200 mg) 


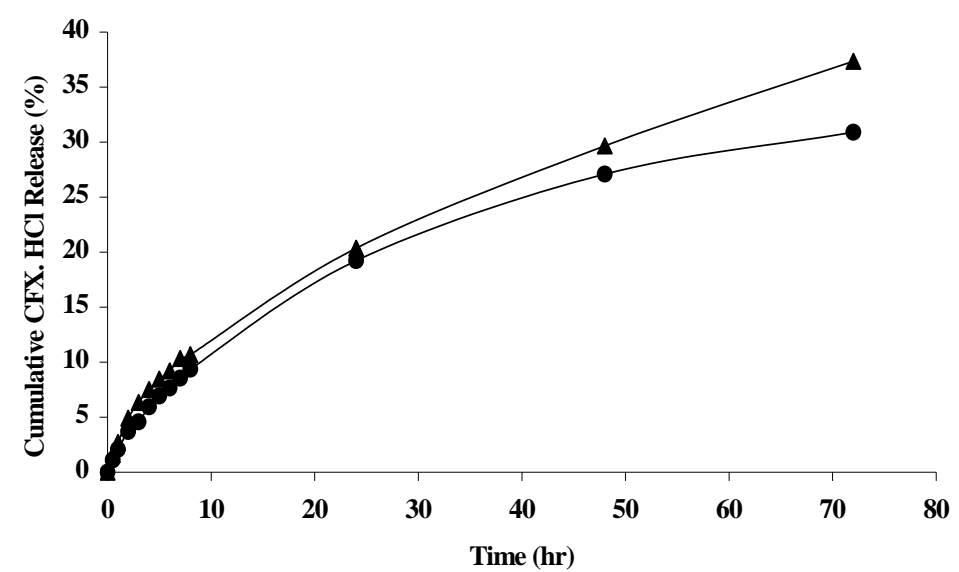

Figure 8: Effect of compression force on the drug release from tablets with chitosan: pectin ratio 1:2.

chains attached to the matrix backbone, and subsequent reduction of tablets compact volume and porosity, that is quite logical to influence drug release rate. Gupta and Bansal $^{36}$ suggested that compression force changes the dimensions of interparticulate voids and modifies the drug-release kinetics. These voids govern both rate of fluid penetration into matrix and release of dissolved drug.

The above results demonstrated that it was possible to produce implantable matrices for a site specific application of antibiotics. $\mathrm{CFX.HCl}$ release can be sustained from matrices containing chitosan: pectin at ratio 1:2. In-vitro drug release was markedly affected by the nature of polyanion polymer, mixing ratio, drug loading, total weight of tablets and to a lesser extent by compression force. The results also showed that such factors can be employed to control the drug release time to the point that it becomes a useful tool in matrix formulation.

\section{Kinetic analysis of the release data}

The kinetic parameters and related rate constants for all tested tablet formulations are shown in Table 2. The drug release data of all prepared tablets showed high linearity with Korsmeyer equation $\left(\mathrm{R}^{2}\right.$ range 0.981 0.999). The values of release exponent " $n$ " determined form the various tablets (F1-F7) showed nonFickian release model $(0.5>\mathrm{n}<1)$ and indicated combined effect of diffusion and polymer chain relaxation mechanisms for drug release. By reviewing the release kinetic data in Table 2, it could be revealed that the drug loading plays an important role on the mechanism of drug release from matrices and a 
possible effect to modulate drug release from tablets. The " $n$ " values for tablets with drug loading $2.5 \%$ (F4), 5\% (F6), 10\% (F7) and 25\% (F8) were $0.89,0.78,0.65$ and 0.56 , respectively, indicating the gradual shift of the drug release mechanism from non-Fickian to be more closer to Fickian diffusion with higher drug concentration.

Table 2 also showed a gradual decrease of the kinetic constant release values " $\mathrm{K}$ " with increasing drug concentration from $2.5 \%$ to $25 \%$ $\mathrm{w} / \mathrm{w}$, indicating that CFX.HCl was more slowly released from tablets with higher drug loading. Tablets containing 25\% CFX.HCl had the smallest $\mathrm{K}$ value $(1.39 \% / \mathrm{h} . \mathrm{n})$ indicating a marked decrease in drug release as compared to the $\mathrm{K}$ values 3.62, 2.93 and 2.95 for tablets with $2.5 \%, 5 \%$ and $10 \% \mathrm{w} / \mathrm{w}$ drug loading, respectively.

The effect of tablets total weight on the mechanism of CFX.HCl release showed that increasing the matrix weight had a marked effect on changing the mechanism of the drug release. The " $\mathrm{n}$ " values were 0.65 and 0.52 and "K values" were 2.95 and 1.35 for $100 \mathrm{mg}$ (F7) and $200 \mathrm{mg}$ (F9) tablets, respectively. Such results revealed a change in the drug release mechanism from non-Fickian to be Fickian with a subsequent decrease in drug diffusion outwards the system.

Table 2: Dissolution and kinetic release parameters of ciprofloxacin hydrochloride tablet matrices.

\begin{tabular}{||c|c|c|c|c|c|c||}
\hline Formula & $\begin{array}{c}\text { \% Drug } \\
\text { Release } \\
\text { after } 8 \mathrm{hrs}\end{array}$ & $\begin{array}{c}\text { Release } \\
\text { Exponent } \\
(\mathrm{n})\end{array}$ & $\begin{array}{c}\text { Kinetic } \\
\text { Constant } \\
(\mathrm{K})\left(\% / \mathrm{h}^{\mathrm{n}}\right)\end{array}$ & $\begin{array}{c}\text { Determination } \\
\text { Coefficient } \\
\left(\mathrm{R}^{2}\right)\end{array}$ & $\begin{array}{c}\text { Mechanism } \\
\text { of Release }\end{array}$ & $\begin{array}{c}\text { MDT } \\
(\mathrm{h})\end{array}$ \\
\hline F1 & 47.64 & 0.92 & 7.23 & 0.996 & Non-Fickian & 0.06 \\
\hline F2 & 42.18 & 0.88 & 6.83 & 0.999 & Non-Fickian & 0.05 \\
\hline F3 & 31.12 & 0.83 & 5.71 & 0.997 & Non-Fickian & 0.06 \\
\hline F4 & 22.26 & 0.89 & 3.62 & 0.999 & Non-Fickian & 0.11 \\
\hline F5 & 20.46 & 0.87 & 3.49 & 0.999 & Non-Fickian & 0.11 \\
\hline F6 & 14.39 & 0.78 & 2.93 & 0.997 & Non-Fickian & 0.11 \\
\hline F7 & 10.69 & 0.65 & 2.95 & 0.987 & Non-Fickian & 0.07 \\
\hline F8 & 4.41 & 0.56 & 1.39 & 0.997 & Closer to Fickian & 0.20 \\
\hline F9 & 4.22 & 0.52 & 1.35 & 0.981 & Closer to Fickian & 0.19 \\
\hline F10 & 9.37 & 0.72 & 2.14 & 0.997 & Non-Fickian & 0.15 \\
\hline
\end{tabular}

CS: Chitosan; CMC: Carboxymethylcellulose; ALG: Alginate; Pe: Pectin MDT: Mean Dissolution Time. 
The effect of compression force on " $n$ " values was investigated. The results showed that with increasing compression pressure from 2 tons (F7) to 5 tons (F10), the " $n$ " values were 0.65 and 0.72 , respectively, indicating non-Fickian mechanism for the drug release. The $\mathrm{K}$ value was decreased from 2.95 to $2.14 \mathrm{~h} .{ }^{\mathrm{n}}$. These results revealed the independence of drug release mechanism from matrix tablets on the applied compression force.

Mean dissolution time (MDT) values are used to characterize the drug release rate and indicate the drug release retarding efficiency of polymeric matrices. Table 2 , showed that MDT values are independent on the polyanion type but dependent on its ratio. Increasing the ratio of chitosan in the matrix (F4 and F5) showed a higher MDT value $(0.11 \mathrm{~h})$ in comparison to $\mathrm{F} 3$ matrix (MDT = $0.06 \mathrm{~h}$ ), thus confirming the effect of chitosan on controlling the drug release. Ten fold increase in drug loading from $2.5 \%(\mathrm{~F} 4)$ to $25 \%(\mathrm{~F} 8)$ and doubling matrix weight from 100 $\mathrm{mg}$ (F7) to $200 \mathrm{mg}$ (F8) showed obvious effect on retarding the drug release from the matrix (MDT $=0.2$ h). Increase compression force from 2 tons (F7) to 5 tons (F10) increased MDT from $0.07 \mathrm{~h}$ to $0.15 \mathrm{~h}$.

\section{Statistical analysis of the drug dissolution data}

The effect of the formulation variables on $\mathrm{CFX} . \mathrm{HCl}$ dissolution profiles was studied for tested matrices (Table 3). The results of polyanion polymers showed a similarity in the release profiles of CMC compared to ALG or Pe $\left(\mathrm{f}_{2}>\right.$ 50) and a slight difference between ALG and Pe $\left(f_{1}=32\right)$. Change in chitosan/pectin ratio showed no difference in their dissolution profiles as $\mathrm{f} 2>50$. An obvious increase in similarity $\left(f_{2}=90\right)$ was observed as the concentration of chitosan increased (CS: Pe 1:2 Vs. 1:1). The effect of drug loading on release profiles for different concentrations ranged from $2.5 \%$ - $25 \%$ showed a marked similarity and high $\mathrm{f}_{2}$ values (57 - 82) for all profiles except the release profile of $2.5 \%$ Vs. $25 \%$ that showed a marked difference $f_{1}=77$. This results also confirming the effect of drug loading on changing the release mechanism from the matrix tablets from the non Fickian to Fickian. Matrix weights and compression force showed no effect on the dissolution, since similar profiles with $f_{2}$ values > 50 were recorded.

\section{Microbiological evaluation of release in a gel simulating in-vivo implantation}

Tablet matrices are designed to be placed in the body where they are surrounded by tissues and extracellular fluids. Agar gel could be used to simulate subcutaneous tissues due to their resemblance to subcutaneous tissues in composition, rheologic nature, and water content. Thus, invitro drug release from implants in an agar gel can be expected to closely simulate in-vivo drug release ${ }^{28}$. 
Table 3: $\mathrm{f} 1$ and $\mathrm{f} 2$ Values as a function of $\mathrm{CFX} . \mathrm{HCl}$ tablets formulation parameters.

\begin{tabular}{|l|l|c|c||}
\hline \multicolumn{1}{|c|}{ Variables } & \multicolumn{1}{|c|}{ Matrix compositions } & $\mathrm{f}_{1}$ & $\mathrm{f}_{2}$ \\
\hline 1- Polyanion polymers & CS: ALG Vs. CS:CMC & 11 & 71 \\
& CS: CMC Vs. CS: Pe & 23 & 58 \\
& CS: ALG Vs CS: Pe & 32 & 49 \\
\hline 2- CS: Pe ratios & $1: 3$ Vs. 1:2 & 30 & 60 \\
& $1: 3$ Vs. $1: 1$ & 36 & 56 \\
& $1: 2$ Vs. 1:1 & 8 & 90 \\
\hline 3- Drug concentrations & $2.5 \%$ Vs. 5\% & 32 & 64 \\
& $2.5 \%$ Vs. 10\% & 45 & 57 \\
& $2.5 \%$ Vs. 25\% & 77 & 46 \\
& $5 \% \quad$ Vs. 10\% & 18 & 82 \\
& $5 \% \quad$ Vs. 25\% & 66 & 58 \\
& $10 \%$ Vs. 25\% & 59 & 66 \\
\hline 4- Tablet weight & $100 \mathrm{mg}$ Vs. 200 mg & 62 & 65 \\
\hline 5- Compression force & 2 tons Vs. 5 tons & 19 & 88 \\
\hline
\end{tabular}

$\left(\mathrm{f}_{2}\right)=(\%)$ similarity between the two curves

$\left(\mathrm{f}_{1}\right)=(\%)$ difference between the two curves

Tablet matrices composed of CS: Pe ratio 1: 2 and drug loading 10\% (F7) and $25 \%$ (F8) were selected to study efficacy and release of CFX.HCl. Measurement of mean inhibition zone of agar plates (Fig. 9) showed the highest bactericidal activity of CFX.HCl against gramnegative bacteria (Escherichia coli) compared to gram-positive (Staphylococcus aureus). Mean diameters of inhibition zone for Escherichia coli were $7.4 \mathrm{~cm}$ and 6.8 $\mathrm{cm}$; Staphylococcus aureus were 5.3 $\mathrm{cm}$ and $4.2 \mathrm{~cm}$ for tablets containing
$10 \%$ and $25 \%$ drug, respectively. The results showed that there is no bacterial growth after $24 \mathrm{hrs}$ with both tablets loading with $10 \%$ and $25 \%$ drug. Extending fermentation time to $48 \mathrm{hrs}$ showed bacterial growth for microorganisms separated from plates containing $10 \%$ drug load; while those separated from plates containing $25 \%$ drug load showed no sign of bacterial growth. CFX . $\mathrm{HCl}$ loading in the suggested matrices resulted in a prolonged release and well-controlled effect on the bacterial growth (F8). 


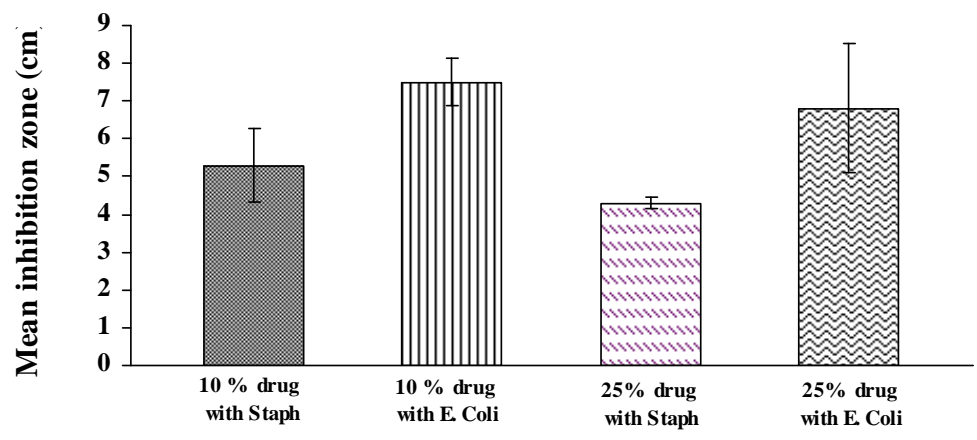

Fig. 9 Mean diameter of inhibition zones of agar gel plates seeded with Staphylococcus aureus and Escherichia coli after implantation of ciprofloxacin loaded matrics in the center of the plates

\section{Conclusion}

An easy and reproducible ciprofloxacin hydrochloride implantable delivery system based on natural biodegradable polymers (chitosan and pectin) was developed. The biodegradable drug delivery systems would have advantage of eliminating the need for additional surgery to remove the carrier. Polyanion complex of chitosan with pectin was formed at weight ratio 1:2. Drug release from tested tablets was influenced by type of polyanion polymer, polymers mixing ratio, drug loading, matrix weight, whereas compression pressure does not affect the drug release significantly. Drug release data of tablet matrices showed high linearity with Korsmeyer equation $\left(\mathrm{R}^{2}\right.$ range 0.981-0.999) and indicated combined effect of diffusion and polymer chain relation mechanisms. Increasing drug loading and tablet weight change the drug release to be more closer to Fickian mechanism. Microbiological simulated test demonstrated the efficacy of chitosan: pectin $(1: 2)$ tablets with $25 \%$ drug load on extending the period of the drug delivery.

Chitosan/ polyanion implantable devices loaded with CFX.HCl could serve as a site-specific delivery systems for bone, tissue infection or prevention of post-surgical wound infection. The convenience of oncedosing implant may potentially result in improved patient compliance and reduced cost of antibiotics.

\section{REFERENCES}

1- L. R. Gill, K. J. MayberryCarson, B. Tober-Meyer, F. Hodin, D. W. Lambe and B. G. Painter, Microbios., 58, 113 (1989).

2- A. Sanchez-Navarro, A. C. Casquero-Dorado and M. Weiss, Pharm. Res., 16, 587 (1999). 
3- R. Davis, A. Markham and J. A. Balfour, Drugs, 51, 1019 (1996).

4- U. R. Kamath, U. V Singh and N. Udupa, Indian J. Pharm. Sci., 55, 148 (1993).

5- Y. Özsoy, T. Tuncel, A. Can and N. Akev, Pharmazie, 55, 607 (2000).

6- K. P. R. Chowdary and P. A. Kumar, Indian J. Pharm. Sci., 58, 47 (1996).

7- B. Martinez, F. Lairion, M. B. Pena, P. D Rocco and M. C. Nacucchio, Microencapsul., 14, 155 (1997).

8- G. Owusu-Ababio and J. Rogers, Eur. J. Pharm. Biopharm., 42, 188 (1996).

9- M. E. Page-Clisson, H. PintoAlphandary, M. Ourevitch, A. Andremont and P. Couvreur, J. Control. Rel., 56, 23 (1998).

10- F. Fawaz, M. Guyot, A. M. Lagueny and J. Ph. Devissaguet, Int. J. Pharm., 154, 191 (1997).

11- A. Gursoy and B. Senyucel, J. Microencapsul., 14, 769 (1997).

12- C. Désévaux, P. Dubreuil and V. Lenaerts, J. Control. Rel., 82, 83 (2002).

13- M. Ramchandani and D. Robinson, ibid., 54, 167 (1998).

14- W. R. Gombotz and S. F. Wee, Adv. Drug Deliv. Rev., 31, 267 (1998).

15- P. Giunchedi, U. Conte, P. Chetoni and M. F. Saettones, Eur. J. Pharm. Sci., 9, 1 (1999).

16- T. W. Wong, L. W. Chan, H. Y. Lee and P. W. Heng, J. Microencapsul., 19, 511 (2002).
17- J. Wang and P. Somasundaran, J. Colloid and Interface Science, 291, 75 (2005).

18- L. Ilium, Pharm. Res., 15, 1326 (1998).

19- S. Hirano, H. Seino, Y. Akiyama and I. Nonaka, Polym. Eng. Sci., 59, 897 (1988).

20- V. L. Gonçalves, M. C. M. laranjeira and V. T. Fávere, Polímeros: Ciência e Tecnologia, 15, 6 (2005).

21- L. L. Balassa and J. F. Prudden, "Applications of Chitin and Chitosan in Wound Healing Acceleration", in: Chitin, Chitosan and Related Enzymes, Academic Press, San Diego, 1984, pp. 296-305.

22- R.A. A. Muzzarelli, "Amphoteric Derivatives of Chitosan and their Biological Significance", in: Chitin and Chitosan, Elsevier Applied Science, London, 1989, pp. 87-99.

23- C. Remunan-Lopez, A. Portero, J. L. Vila-Jato and M. J. Alonso, J. Control. Rel., 55, 143 (1998).

24- R. W. Korsmeyer, R. Gurny, E. Docler, P. Buri and N. A. Peppas, Int. J. Pharm., 15, 25 (1983).

25- N. A. Peppas, Pharm. Acta. Helv., 60, 110 (1985).

26- J. E. Mockel and B. C. Lippold, Pharm. Res., 10, 1066 (1993).

27- J. W. Moore and H. H. Flanner, Pharmaceutical Technology, 20, 6, 64 (1996).

28- S. Allababidi and J. C Shah, J. Pharm. Sci., 87, 738 (1998). 
29- G. S. Macleod, J. H. Collett and J. T. Fell, J. Control. Rel., 58, 303 (1999).

30- Y. Sawayanagi, N. Nambu and T. Nagai, Chem. Pharm. Bull., 30, 3297 (1982).

31- K. D. Yao, H. Tu, C. Fa, J. W. Zhang and J. Liu, Angew. Makromol. Chem., 245, 63 (1997).

32- J. A. Hunt, G. Meijs and D. F. Williams, J. Biomed. Mater. Res., 36, 542 (1997).
33- K. L. B. Chang and J. Lin, Carbohydrate Polymers, 43, 163 (2000).

34- A. Ghaffari, K. Navaee, M. Oskoui, K. Bayati and M. R.Tehrani, Eur. J. Pharm. and Biopharm., 67, 175 (2007).

35- J. Mulhbacher, P. Ispas-Szabo, V. Lenaerts and M. A. Mateescu, J. Control. Rel., 76, 51 (2001).

36- P. Gupta and A. K. Bansal, Pharm. Tech. Eur., 10, 47 (2002). 